\title{
About Some Families of Nonexpansive Mappings with respect to Renorming
}

\author{
Juan Rafael Acosta-Portilla, Carlos Alberto Hernández-Linares, and Víctor Pérez-García
}

Facultad de Matemáticas, Universidad Veracruzana, Circuito Gonzalo Aguirre Beltrán S/N, Zona Universitaria, 91090 Xalapa, VER, Mexico

Correspondence should be addressed to Carlos Alberto Hernández-Linares; carlhernandez@uv.mx

Received 5 September 2016; Accepted 25 October 2016

Academic Editor: Filomena Cianciaruso

Copyright (C) 2016 Juan Rafael Acosta-Portilla et al. This is an open access article distributed under the Creative Commons Attribution License, which permits unrestricted use, distribution, and reproduction in any medium, provided the original work is properly cited.

We characterize the family of nonexpansive mappings which are invariant under renormings and we also compare the families of nonexpansive mappings under two equivalent norms.

\section{Introduction}

A Banach space $(X,\|\cdot\|)$ satisfies the fixed point property (FPP) if every nonexpansive mapping $T: C \rightarrow C$ has a fixed point, where $C$ is a closed convex bounded subset of $X$. For a long time, it was an open problem if the fixed point property could be equivalent to the reflexivity. It was until 2008, when Lin [1] proved that there exists a nonreflexive Banach space with the FPP. Actually, Lin used a renorming $\|\cdot\|_{L}$ of the space $\ell_{1}$ such that $\left(\ell_{1},\|\cdot\|_{L}\right)$ has the FPP. One year later, Domínguez-Benavides proved that every reflexive Banach space can be renormed to have the FPP [2]. After these two articles, the Fixed Point Property and Renorming Theory were clearly connected. However, not all nonreflexive Banach spaces can be renormed to have the FPP as it was proved in [3]; in this work it is proved that $\ell_{\infty}$ and $\ell_{1}(\Gamma)$ where $\Gamma$ is an uncountable set cannot be renormed to have the FPP. Recently, many works have appeared to be looking for new examples of nonreflexive Banach spaces enjoying the FPP or trying to find some structure on families of equivalent norms with the FPP. In the first sense the works should be mentioned $[4-7]$. In the second way the works are remarkable $[8,9]$. After Lin's result, the question remains open: does reflexivity implies FPP? In this setting the relevant paper is [10]. The FPP under a renorming also has been studied for other kinds of mappings; for example, see [11, 12].
From the papers cited in the previous paragraph the FPP is not an isomorphic property. Moreover, the family of nonexpansive mappings could be changed after a renorming. For this reason, the aim of this paper is to say something about the following question.

What happened with the family of nonexpansive mappings with another equivalent norm?

In order to do that, we make the following assumptions. Let $(X,\|\cdot\|)$ be a normed space and $C$ a nonempty subset of $X$; then for each $\|\cdot\|$-Lipschitz function $T: C \rightarrow C$ we denote by $K(T,\|\cdot\|)$ its Lipschitz constant with respect to $\|\cdot\|$ and by $\mathfrak{N}(X)$ the collection of equivalent norms on $X$. For each $\|\cdot\|_{0}$ norm on $X$ we define

$$
N E\left(C,\|\cdot\|_{0}\right)=\left\{S: C \longrightarrow C \mid K\left(S,\|\cdot\|_{0}\right) \leq 1\right\} .
$$

\section{The Family of Nonexpansive Mappings over Every Renorming}

In this section we will study some families of nonexpansive mappings over every renorming and we will characterize them. The first approximation is to characterize the set $\mathcal{S}^{\prime}(C)=\bigcap_{\|\cdot\| \in \Re(X)} N E(C,\|\cdot\|)$, when $C$ is a convex, closed, and bounded set in $X$. It is worthwhile to mention that we prove that at least there are many elements in $N E(C,\|\cdot\|)$ as elements in $N E([0,1],|\cdot|)$. Moreover, we show that their structure is similar. 
For each $x \in C$ we call $f_{x}: C \rightarrow C$ the constant function $x$ and we denote by $I: C \rightarrow C$ the identity map. We define

$$
\mathcal{S}(C)=\operatorname{conv}\left(\left\{f_{x} \mid x \in C\right\} \cup\{I\}\right) .
$$

Remark 1. It is clear that, for each norm $\|\cdot\|$ on $X, T \in$ $N E(C,\|\cdot\|)$ for all $T \in \mathcal{S}(C)$. It is easy to prove that each element $T \in \mathcal{S}(C)$ is of the form $T=y+\alpha I$, for some $y \in X$ and $0 \leq \alpha \leq 1$.

In the rest of this paper we will denote by $\mathbb{F}$ the scalar field $\mathbb{R}$ or $\mathbb{C}$, associated with the normed space. In order to characterize the set $\mathcal{S}^{\prime}(C)$ we will give the following theorem.

Theorem 2. Let $C$ be a nonempty subset of a normed space $X$ and $T: C \rightarrow C$; then the next statements are equivalent.

(1) For each norm $\|\cdot\|$ on $X$ one has that $T \in N E(C,\|\cdot\|)$.

(2) For each $\|\cdot\| \in \mathfrak{N}(X)$ one has that $T \in N E(C,\|\cdot\|)$.

(3) $\|\cdot\|_{0} \in \mathfrak{N}(X)$ exists such that $T \in N E\left(C,\|\cdot\|_{0}\right)$ and for each $x, y \in C, T x-T y \in \operatorname{span}\{x-y\}$ holds.

(4) For each $x, y \in C \alpha \in \mathbb{F}$ exists with $|\alpha| \leq 1$ such that $\alpha(x-y)=T x-T y$.

Proof. It is straightforward that (1) implies (2).

We are going to prove that (2) implies (3); in order to do this we proceed by contraposition. We may suppose that $\|\cdot\|_{0} \in \mathfrak{N}(X)$ exist such that $T \in N E\left(C,\|\cdot\|_{0}\right)$ and $x, y \in C$ such that $T x-T y \notin \operatorname{span}\{x-y\}$.

Since $T x-T y \notin \operatorname{span}\{x-y\}$ then $Y=\operatorname{span}\{x-y, T x-T y\}$ has dimension 2 and then a projection $P$ from $X$ to $Y$ exists that is $\|\cdot\|_{0}$-bounded. Since $Y$ is finite and dimensional and the set $\{x-y, T x-T y\}$ is linear and independent, we can construct a norm $\|\cdot\|_{1}$ in $Y$ such that $\|x-y\|_{1}<\|T x-T y\|_{1}$; note that $\|T x-T y\|_{1}-\|x-y\|_{1}$ could be as large as we desire. We consider the function $\|\cdot\|_{2}$ defined in $X$ by $\|x\|_{2}=\|P x\|_{1}+\|(I-P) x\|_{0}$; it is easy to check that $\|\cdot\|_{2}$ is a norm on $X$. Now we will prove that $\|\cdot\|_{2}$ is equivalent to $\|\cdot\|_{0}$. Since $\|\cdot\|_{0}$ and $\|\cdot\|_{1}$ are equivalent in $Y$ then $0<l \leq u$ the optimal constants exist such that

$$
l\|a\|_{0} \leq\|a\|_{1} \leq u\|a\|_{0} \quad \text { for each } a \in Y .
$$

Since $P$ is $\|\cdot\|_{0}$-bounded, we have for each $z \in X$

$$
\begin{aligned}
\|z\|_{2} & =\|P z\|_{1}+\|(I-P) z\|_{0} \leq u\|P z\|_{0}+\|(I-P) z\|_{0} \\
& \leq(u\|P\|+\|I-P\|)\|z\|_{0} .
\end{aligned}
$$

On the other hand we have

$$
\|z\|_{2}=\|P z\|_{1}+\|(I-P) z\|_{0} \geq l\|P z\|_{0}+\|(I-P) z\|_{0} .
$$

There are two possibilities, $l \geq 1$ or $l<1$. If $l \geq 1$ then

$$
\|z\|_{2} \geq\|P z\|_{0}+\|(I-P) z\|_{0} \geq\|z\|_{0} .
$$

If $l<1$ then

$$
\begin{aligned}
\|z\|_{2} & \geq l\|P z\|_{0}+l\|(I-P) z\|_{0} \\
& =l\left(\|P z\|_{0}+\|(I-P) z\|_{0}\right) \geq l\|z\|_{0} ;
\end{aligned}
$$

thus $\|\cdot\|_{2}$ is equivalent to $\|\cdot\|_{0}$.
Now we have

$$
\begin{aligned}
\|x-y\|_{2} & =\|P(x-y)\|_{1}+\|(I-P)(x-y)\|_{0} \\
& =\|x-y\|_{1}<\|T x-T y\|_{1} \\
& =\|P(T x-T y)\|_{1}+\|(I-P)(T x-T y)\|_{0} \\
& =\|T x-T y\|_{2} ;
\end{aligned}
$$

that is, $T \notin N E\left(C,\|\cdot\|_{2}\right)$.

Now we prove that (3) implies (4). Let $x, y \in C$; if $T x=T y$ the proof is over because $\alpha=0$ is the required constant; then without loss of generality we may assume that $T x \neq T y$; hence $x \neq y$ and since $T x-T y \in \operatorname{span}\{x-y\}$ then $\alpha \in \mathbb{F}$ exist such that $\alpha(x-y)=T x-T y$; thus

$$
\|x-y\|_{0} \geq\|T x-T y\|_{0}=|\alpha|\|x-y\|_{0} .
$$

Therefore $|\alpha| \leq 1$.

Now we show that (4) implies (1). Let $\|\cdot\|$ be a norm on $X$ and $x, y \in C$; then $\alpha \in \mathbb{F}$ exists with $|\alpha| \leq 1$ such that $\alpha(x-y)=T x-T y$; therefore

$$
\|x-y\| \geq|\alpha|\|x-y\|=\|T x-T y\| .
$$

Thus $T \in N E(C,\|\cdot\|)$.

Remark 3. Note that $K\left(T,\|\cdot\|_{2}\right)$ could be arbitrary large, since in the proof of (2) implying (3) in the previous theorem we can take $\|T x-T y\|_{1}-\|x-y\|_{1}$ as large as we want.

Lemma 4. Let $C$ be a nonempty subset of a normed space, $T$ : $C \rightarrow C$, and $x, y, z \in C$ are distinct such that, for each $\alpha \in \mathbb{F}$, and $z \neq \alpha x+(1-\alpha) y, \alpha_{x, y}, \alpha_{x, z}, \alpha_{y, z} \in \mathbb{F}$ exist with

$$
\begin{gathered}
\alpha_{x, y}(x-y)=T x-T y, \\
\alpha_{x, z}(x-z)=T x-T z, \\
\alpha_{y, z}(y-z)=T y-T z ;
\end{gathered}
$$

then $\alpha_{x, y}=\alpha_{x, z}=\alpha_{y, z}$.

Proof. From (11) it follows that

$$
\alpha_{y, z}(y-z)=\alpha_{x, z}(x-z)-\alpha_{x, y}(x-y)
$$

Let $\operatorname{span}_{\mathbb{R}}\{x-y, x-z\}=\left\{r_{1}(x-y)+r_{2}(x-z) \mid r_{1}, r_{2} \in \mathbb{R}\right\}$. Since the set $\{x-y, x-z\}$ is linearly independent, then we can define the operator $R: \operatorname{span}\{x-y, x-z\} \rightarrow \operatorname{span}_{\mathbb{R}}\{x-y, x-z\}$ by $R(\alpha(x-y)+\beta(x-z))=\operatorname{Re}(\alpha)(x-y)+\operatorname{Re}(\beta)(x-z)$ for each $\alpha, \beta \in \mathbb{F}$. It is clear that $R$ is a real linear projection,

$$
R(\alpha w)=\operatorname{Re}(\alpha) R(w)
$$

for each $\alpha \in \mathbb{F}, w \in \operatorname{span}_{\mathbb{R}}\{x-y, x-z\}$, 
and $y-z \in \operatorname{span}_{\mathbb{R}}\{x-y, x-z\}$. For this and (12) we have

$$
\begin{aligned}
\operatorname{Re}\left(\alpha_{y, z}\right)(y-z)= & \operatorname{Re}\left(\alpha_{y, z}\right) R(y-z) \\
= & R\left(\alpha_{y, z}(y-z)\right) \\
= & R\left(\alpha_{x, z}(x-z)-\alpha_{x, y}(x-y)\right) \\
= & \operatorname{Re}\left(\alpha_{x, z}\right)(x-z) \\
& -\operatorname{Re}\left(\alpha_{x, y}\right)(x-y) .
\end{aligned}
$$

We define the linear operator $\phi: \operatorname{span}_{\mathbb{R}}\{x-y, x-z\} \rightarrow \mathbb{R}^{2}$ by $\phi(x-y)=(1,0)=e_{1}$ and $\phi(x-z)=(0,1)=e_{2}$; then

$$
\begin{aligned}
& \operatorname{Re}\left(\alpha_{x, y}\right) e_{1}=\phi\left(\operatorname{Re}\left(\alpha_{x, y}\right)(x-y)\right), \\
& \operatorname{Re}\left(\alpha_{x, z}\right) e_{2}=\phi\left(\operatorname{Re}\left(\alpha_{x, z}\right)(x-z)\right) ;
\end{aligned}
$$

thus

$$
\begin{aligned}
\operatorname{Re} & \left(\alpha_{x, z}\right) e_{2}-\operatorname{Re}\left(\alpha_{x, y}\right) e_{1} \\
& =\phi\left(\operatorname{Re}\left(\alpha_{x, z}\right)(x-z)-\operatorname{Re}\left(\alpha_{x, y}\right)(x-y)\right) \\
& =\phi\left(\operatorname{Re}\left(\alpha_{y, z}\right)(y-z)\right) \\
& =\phi\left(\operatorname{Re}\left(\alpha_{y, z}\right)[(x-z)-(x-y)]\right) \\
& =\operatorname{Re}\left(\alpha_{y, z}\right)\left(e_{2}-e_{1}\right) .
\end{aligned}
$$

We consider the triangles in $\mathbb{R}^{2}$ :

$$
\begin{gathered}
\triangle 0 e_{1} e_{2}, \\
\triangle 0 \operatorname{Re}\left(\alpha_{x, y}\right) e_{1} \operatorname{Re}\left(\alpha_{x, z}\right) e_{2} .
\end{gathered}
$$

Since

$$
\begin{gathered}
\operatorname{Re}\left(\alpha_{x, y}\right) e_{1} \in \operatorname{span}_{\mathbb{R}}\left\{e_{1}\right\}, \\
\operatorname{Re}\left(\alpha_{x, z}\right) e_{2} \in \operatorname{span}_{\mathbb{R}}\left\{e_{2}\right\}, \\
\operatorname{Re}\left(\alpha_{x, z}\right) e_{2}-\operatorname{Re}\left(\alpha_{x, y}\right) e_{1} \in \operatorname{span}\left\{e_{2}-e_{1}\right\},
\end{gathered}
$$

then the triangles are similar; thus

$$
\operatorname{Re}\left(\alpha_{x, y}\right)=\operatorname{Re}\left(\alpha_{x, z}\right)=\operatorname{Re}\left(\alpha_{y, z}\right) .
$$

In a similar way we prove that $\operatorname{Im}\left(\alpha_{x, y}\right)=\operatorname{Im}\left(\alpha_{x, z}\right)=$ $\operatorname{Im}\left(\alpha_{y, z}\right)$, by considering the operator $R(\alpha(x-y)+\beta(x-z))=$ $\operatorname{Im}(\alpha)(x-y)+\operatorname{Im}(\beta)(x-z)$.

Then $\alpha_{x, y}=\alpha_{x, z}=\alpha_{y, z}$.

Theorem 5. Under assumptions of Theorem 2 and if $C$ has at most two elements or for each distinct $x, y \in C z \in C$ exists such that, for each $\alpha \in \mathbb{F}, z \neq \alpha x+(1-\alpha) y$, then the statement,

(A) $T=y+\alpha I$ for some $\alpha \in \mathbb{F}$ with $|\alpha| \leq 1$ and $y \in X$, is equivalent to each statement in that theorem.
Proof. The statement (A) implies that (1) in Theorem 2 is obvious.

Now we show that (4) of Theorem 2 implies (A). If $C=\{x\}$ then $T x=0+1 I x$; if $C=\{x, y\}$ with $x \neq y$ and $\alpha$ is such that $\alpha(x-y)=T x-T y$ then $T z=w+\alpha I z$ with $w=T y-\alpha y$ for each $z \in C$. We suppose that for each distinct $x, y \in C z \in C$ exists such that, for each $\alpha \in \mathbb{F}, z \neq \alpha x+(1-\alpha) y$. For each $x, y \in C$ let $\alpha_{x, y} \in \mathbb{F}$ such that

$$
\alpha_{x, y}(x-y)=T x-T y .
$$

It is clear that $\alpha_{x, y}=\alpha_{y, x}$. Let $x, y \in C$ with $x \neq y$. It is enough to prove that, for each distinct $z_{1}, z_{2} \in C, \alpha_{z_{1}, z_{2}}=\alpha_{x, y}$. Let $z_{1}, z_{2} \in C$; we may suppose without loss of generality that the following cases are exhaustive.

(i) $\alpha_{1}, \alpha_{2} \in \mathbb{F}$ exist such that

$$
\begin{aligned}
& z_{1}=\alpha_{1} x+\left(1-\alpha_{1}\right) y, \\
& z_{2}=\alpha_{2} x+\left(1-\alpha_{2}\right) y .
\end{aligned}
$$

(ii) $\alpha_{1} \in \mathbb{F}$ exists such that

$$
z_{1}=\alpha_{1} x+\left(1-\alpha_{1}\right) y
$$

and for each $\alpha_{2} \in \mathbb{F}$

$$
z_{2} \neq \alpha_{2} x+\left(1-\alpha_{2}\right) y
$$

(iii) For each $\alpha_{1}, \alpha_{2} \in \mathbb{F}$

$$
\begin{aligned}
& z_{1} \neq \alpha_{1} x+\left(1-\alpha_{1}\right) y, \\
& z_{2} \neq \alpha_{2} x+\left(1-\alpha_{2}\right) y .
\end{aligned}
$$

We assume (i) and $z_{1} \neq x$; then by hypothesis $z \in C$ exist such that for each $\alpha \in \mathbb{F}$

$$
\begin{aligned}
& z \neq \alpha x+(1-\alpha) y, \\
& z \neq \alpha x+(1-\alpha) z_{1}, \\
& z \neq \alpha z_{1}+(1-\alpha) z_{2} ;
\end{aligned}
$$

then by Lemma 4

$$
\begin{gathered}
\alpha_{x, y}=\alpha_{x, z}=\alpha_{z, y}, \\
\alpha_{x, z_{1}}=\alpha_{x, z}=\alpha_{z, z_{1}}, \\
\alpha_{z_{1}, z_{2}}=\alpha_{z_{1}, z}=\alpha_{z, z_{2}} .
\end{gathered}
$$

Thus $\alpha_{x, y}=\alpha_{x, z}=\alpha_{z, z_{1}}=\alpha_{z_{1}, z_{2}}$.

We suppose (ii), $z_{1} \neq x$, and $z_{1} \neq y$; then for each $\alpha \in \mathbb{F}$

$$
z_{2} \neq \alpha x+(1-\alpha) z_{1}
$$

thus by Lemma 4

$$
\begin{gathered}
\alpha_{x, y}=\alpha_{x, z_{2}}=\alpha_{z_{2}, y}, \\
\alpha_{x, z_{1}}=\alpha_{x, z_{2}}=\alpha_{z_{2}, z_{1}} .
\end{gathered}
$$

Then $\alpha_{z_{1}, z_{2}}=\alpha_{x, y}$. 
We suppose (iii) and we may assume that for each $\alpha \in \mathbb{F}$

$$
x \neq \alpha z_{1}+(1-\alpha) z_{2}
$$

then by Lemma 4

$$
\begin{gathered}
\alpha_{x, y}=\alpha_{x, z_{1}}=\alpha_{z_{1}, y} \\
\alpha_{z_{1}, z_{2}}=\alpha_{z_{1}, x}=\alpha_{x, z_{2}}
\end{gathered}
$$

Then $\alpha_{z_{1}, z_{2}}=\alpha_{x, z_{1}}=\alpha_{x, y}$; hence $\alpha_{z_{1}, z_{2}}=\alpha_{x, y}$ for each distinct $z_{1}, z_{2} \in C$.

Remark 6. If $C$ is a nonempty subset of a normed space ( $X$, $\|\cdot\|)$, in such a way that it lies inside a one-dimensional affine subspace of $X$, then $D \subset \mathbb{F}$ and a surjective isometry $\phi:(C$, $\|\cdot\|) \rightarrow(D,|\cdot|)$ exist. If,additionally, $C$ is convex, then $\phi$ can be affine. To prove this, we may suppose that $C$ has at least two points. Let $x, y \in C$ with $x \neq y$ and $s=\|x-y\|$; then we define $\phi:(C,\|\cdot\|) \rightarrow(\mathbb{F},|\cdot|)$ by $\phi(z)=\alpha_{z} s$ where $\alpha_{z}$ is the unique element in $\mathbb{F}$ such that $z=\alpha_{z} x+\left(1-\alpha_{z}\right) y$; it is not hard to check that $\phi$ is a surjective isometry from $C$ to $D=\phi(C)$, and if $C$ is convex, then $\phi$ is affine.

It is not hard to prove that the set $D$ and the isometry $\phi$ can be constructed independent of the norm in $C$; for this it is sufficient to consider the renorming $r|\cdot|$ on $\mathbb{F}$ with $r=\|x-y\|$, $\phi: C \rightarrow \mathbb{F}$ defined by $\phi(z)=\alpha_{z}$, and $D=\phi(C)$.

Theorem 7. Under assumptions of Theorem 2 and if $x, y \in C$ exist such that for each $z \in C \alpha \in \mathbb{F}$ exists with $z=\alpha x+(1-$ $\alpha) y$, then (1) to (4) in Theorem 2 are equivalent to each of the following statements.

(B) For each norm $\|\cdot\|$ on $X, D \subset \mathbb{F}, f \in N E(\mathbb{F},|\cdot|)$, and a surjective isometry $\phi:(C,\|\cdot\|) \rightarrow(D,|\cdot|)$ exist with $T=\left.\phi^{-1} f\right|_{D} \phi$.

(C) $D \subset \mathbb{F}$, a surjective function $\phi: C \rightarrow D$, and $f \in$ $N E(\mathbb{F},|\cdot|)$ exist with $T=\left.\phi^{-1} f\right|_{D} \phi$, and for each norm $\|\cdot\|$ on $X, r|\cdot| \in \mathfrak{N}(\mathbb{F})$ exist such that $\phi$ is a $\|\cdot\|$ to $r|\cdot|$-isometry.

Proof. First we prove that (B) implies (1) of Theorem 2. Let $\|\cdot\|$ be a norm on $X$; then $D \subset \mathbb{F}, f \in N E(\mathbb{F},|\cdot|)$, and a surjective isometry $\phi:(C,\|\cdot\|) \rightarrow(D,|\cdot|)$ exist with $T=\left.\phi^{-1} f\right|_{D} \phi$ and $\left.f\right|_{D}=\phi T \phi^{-1}$; it is not hard to prove that $T$ is a $\|\cdot\|$ Lipschitz function with $K(T,\|\cdot\|) \leq K(f,|\cdot|) \leq 1$; therefore $T \in N E(C,\|\cdot\|)$.

Now we prove that (1) of Theorem 2 implies (B). Let $\|\cdot\|$ be a norm on $X$ and $x, y \in C$ such that for each $z \in C \alpha_{z} \in \mathbb{F}$ exist with $z=\alpha_{z} x+\left(1-\alpha_{z}\right) y$; by Remark $6 D \subset \mathbb{F}$ and a surjective isometry $\phi:(C,\|\cdot\|) \rightarrow(D,|\cdot|)$ exist; we define $g=\phi T \phi^{-1}$; thus $K(g,|\cdot|)=K(T,\|\cdot\|) \leq 1$ and by Kirszbraun's Theorem $[13,14]$, an extension $f:(\mathbb{F},|\cdot|) \rightarrow(\mathbb{F},|\cdot|)$ of $g$ exists such that $K(f,|\cdot|)=K(g,|\cdot|) \leq 1$.

We prove that (B) implies (C). By Remark $6 D \subset \mathbb{F}$ and a surjective $\phi: C \rightarrow D$ exist such that, for each norm $\|\cdot\|$ on $X, r|\cdot| \in \mathfrak{N}(\mathbb{F})$ exists so that $\phi:(C,\|\cdot\|) \rightarrow(D, r|\cdot|)$ is an isometry. We define $g=\phi T \phi^{-1}$; by (A) we have that $T \in N E(C,\|\cdot\|)$; thus $g \in N E(D, r|\cdot|)$. Since the Lipschitz constant of a function defined from a subset of $\mathbb{F}$ to itself is independent of the norm on $\mathbb{F}$, then $g \in N E(D,|\cdot|)$. Hence again by Kirszbraun's Theorem, an extension $f:(\mathbb{F},|\cdot|) \rightarrow$ $(\mathbb{F},|\cdot|)$ exists with $K(f,|\cdot|)=K(g,|\cdot|) \leq 1$.

Now we prove that (C) implies (B). Let $\|\cdot\|$ be a norm on $X$; then $r|\cdot| \in \mathfrak{N}(\mathbb{F})$ exist such that $\phi$ is a $\|\cdot\|$ to $r|\cdot|$ isometry. We define $D^{\prime}=r D, \psi=r \phi$, and $g=\psi T \psi^{-1}$; it is clear that $\psi:(C,\|\cdot\|) \rightarrow\left(D^{\prime},|\cdot|\right)$ is an isometry; thus $K(g,|\cdot|)=K(T,\|\cdot\|) \leq 1$; then again by Kirszbraun's Theorem the conclusion follows.

Corollary 8. Let $(X,\|\cdot\|)$ be a normed space and $C$ a nonempty, convex, closed, and bounded subset of $X$; then

(1) if $x, y \in C$ exist such that for each $z \in C \alpha \in \mathbb{F}$ exists with $z=\alpha x+(1-\alpha) y$, then $D \subset \mathbb{F}$ and a surjective isometry $\phi:(C,\|\cdot\|) \rightarrow(D,|\cdot|)$ exist in such a way that for each $T \in \mathcal{S}^{\prime}(C) f \in N E(D,|\cdot|)$ exist with $K(f,|\cdot|)=K(T,\|\cdot\|)$ and $T=\phi^{-1} f \phi ;$

(2) if for each $x, y \in C z \in C$ exists such that, for each $\alpha \in \mathbb{F}, z \neq \alpha x+(1-\alpha y)$, then $\mathcal{S}^{\prime}(C)=\{T: C \rightarrow C \mid$ $T=y+\alpha I$ for some $y \in X$ and $|\alpha| \leq 1\}$.

In general $\mathcal{S}^{\prime}(C)$ is not equal to $\mathcal{S}(C)$; for instance we take $C=[-1,1]$ with the absolute value norm and $T$ : $[-1,1] \rightarrow[-1,1]$ the operator defined by $T x=-x$; then by Remark $1 T \notin \mathcal{S}(C)$ and by Corollary $8 T \in \mathcal{S}^{\prime}(C)$.

Remark 9. It is important to note that if $C$ is convex, closed, and bounded, then each element in $S^{\prime}(C)$ has a fixed point; to check this we consider the following two cases.

(1) If $C$ lies inside a one-dimensional affine subspace of $X$, then by Schauder's Theorem the conclusion is clear.

(2) We suppose that for each $x, y \in C z \in C$ exists such that, for each $\alpha \in \mathbb{F}, z \neq \alpha x+(1-\alpha) y$. Let $T \in \mathcal{S}^{\prime}(C)$; then by Theorem $5 T x=y+\alpha x$ for some $\alpha \in \mathbb{F}_{\text {with }}$ $|\alpha| \leq 1$ and $y \in X$; then $z \in C$ exists such that $\{z, y\}$ is linearly independent; let $Y=\operatorname{span}\{z, y\}$ and $C_{1}=$ $Y \cap C$; it is not hard to prove that $T\left(C_{1}\right) \subset C_{1}$; thus again by Schauder's Theorem, $\left.T\right|_{C_{1}}$ has a fixed point.

Corollary 10. Let $(X,\|\cdot\|)$ be a normed space and $C$ a nonempty, convex, closed, and bounded subset of $X$ such that for each $x, y \in C z \in C$ exist with $z \neq \alpha x+(1-\alpha) y$; then for each $T \in N E(C,\|\cdot\|) \backslash S^{\prime}(C),\|\cdot\|_{1} \in \mathfrak{N}(X)$ exists with $T \notin N E\left(C,\|\cdot\|_{1}\right)$.

In particular if $T$ is a nonlinear operator or an operator without fixed points, then a renorming $\|\cdot\|_{1}$ of $X$ exists in such a way that $T$ is not a $\|\cdot\|_{1}$-nonexpansive operator and by Remark $3, K\left(T,\|\cdot\|_{1}\right)$ is large as one desires.

Theorem 11. Under assumptions of Theorem 2, the following statements are equivalent to each statement in that theorem.

(D) For each of the norms $\|\cdot\|$ and $\|\cdot\|_{1}$ on $X, K(T,\|\cdot\|)=$ $K\left(T,\|\cdot\|_{1}\right) \leq 1$.

(E) For each $\|\cdot\|,\|\cdot\|_{1} \in \mathfrak{N}(X), K(T,\|\cdot\|)=K\left(T,\|\cdot\|_{1}\right) \leq 1$.

Proof. It is clear that (D) implies (E); now we prove that (E) implies (3) of Theorem 2. For this we proceed by 
contraposition; hence we may suppose that $\|\cdot\| \in \mathfrak{N}(X)$ exist with $K(T,\|\cdot\|) \leq 1$ and $x, y \in C$ such that $T x-T y \notin$ $\operatorname{span}\{x-y\}$; there exists $\|\cdot\|_{1} \in \mathfrak{N}(X)$ with $K\left(T,\|\cdot\|_{1}\right)>1$, by using Remark 3 and similar arguments of the proof of (2) implying (3) in Theorem 2.

We prove that (3) of Theorem 2 implies (D). We have the following two cases.

(i) For each $x, y \in C z \in C$ exist such that, for each $\alpha \in \mathbb{F}$, $z \neq \alpha x+(1-\alpha) y$.

(ii) $x, y \in C$ exist such that for each $z \in C \alpha \in \mathbb{F}$ exist with $z=\alpha x+(1-\alpha) y$.

We suppose (i); then by Theorem 5, $T=y+\alpha I$ for some $y \in X$ and $|\alpha| \leq 1$; thus for each norm the Lipschitz constant of $T$ is $|\alpha|$.

Now we assume (ii); then by (C) in Theorem 7 and the fact that the Lipschitz constant of functions defined from subsets of $\mathbb{F}$ to itself is independent of the norm on $\mathbb{F}$, we have $K(T$, $\|\cdot\|)=K\left(T,\|\cdot\|_{1}\right) \leq 1$ for each of the norms $\|\cdot\|$ and $\|\cdot\|_{1}$ on $X$.

\section{Comparing Families of Nonexpansive Mappings}

Now, we will compare the families of nonexpansive mappings by given conditions in which the families are different. Before that, to prove the two theorems in this section we need to prove two technical lemmas.

Definition 12. Let $X$ be vectorial space endowed with two norms $\|\cdot\|_{1}$ and $\|\cdot\|_{2}$ and $C$ a subset of $X$. One will say that $\|\cdot\|_{1}$ is collinear to $\|\cdot\|_{2}$ in $C$, if there exists $\lambda>0$ such that $\|x\|_{1}=\lambda\|x\|_{2}$ for all $x \in C$. We can omit the subset $C$ if the context is clear.

Remark 13. It is clear that the collinearity between norms is an equivalent relation. Let $T: C \rightarrow C$; note that $K\left(T,\|\cdot\|_{1}\right)=$ $K\left(T,\|\cdot\|_{2}\right)$ if $\|\cdot\|_{1}$ is collinear to $\|\cdot\|_{2}$ in $C-C$.

Lemma 14. Let $X$ be a normed space. If $C$ is a nontrivial convex subset of $X$ with $0 \in C$ and $\|\cdot\|_{1}$ and $\|\cdot\|_{2}$ are two norms over $X$ such that they are not collinear in $C$, then $x, y \in C \backslash\{0\}$ exist such that $\|x\|_{1}=\|y\|_{1}$ and $\|y\|_{2}>\|x\|_{2}$.

Proof. Since $\|\cdot\|_{1}$ and $\|\cdot\|_{2}$ are not collinear in $C$, then it is false that $\lambda>0$ exists such that for all $z \in C$ we have $\|z\|_{1}=\lambda\|z\|_{2}$; that is, the function $f(z)=\|z\|_{1} /\|z\|_{2}$ defined on $C \backslash\{0\}$ is not constant; thus $x^{\prime}, y^{\prime} \in C \backslash\{0\}$ exist such that $\left\|x^{\prime}\right\|_{1} /\left\|x^{\prime}\right\|_{2}>$ $\left\|y^{\prime}\right\|_{1} /\left\|y^{\prime}\right\|_{2}$.

If $\left\|x^{\prime}\right\|_{1}=\left\|y^{\prime}\right\|_{1}$ then we concluded the proof. Else, we call $x$ the element in $\left\{x^{\prime}, y^{\prime}\right\}$ such that $\|x\|_{1}=\min \left\{\left\|x^{\prime}\right\|_{1},\left\|y^{\prime}\right\|_{1}\right\}$; if $x=x^{\prime}$ then we take $y=\left(\|x\|_{1} /\left\|y^{\prime}\right\|_{1}\right) y^{\prime}$, so $\|y\|_{1}=\|x\|_{1}$; since $C$ is convex and $0 \in C$ we have that $y \in C$. Therefore $\|x\|_{1}=\|y\|_{1}$ and $\|y\|_{1} /\|y\|_{2}=\left\|y^{\prime}\right\|_{1} /\left\|y^{\prime}\right\|_{2}$; thus $\|x\|_{2}<\|y\|_{2}$.

If $x=y^{\prime}$ the proof is similar.

Lemma 15. Let $X$ be a normed space, $C$ a nonempty subset of $X,\|\cdot\|_{1}$ and $\|\cdot\|_{2}$ norms over $X$; then the following statements are equivalent.
(1) $\|\cdot\|_{1}$ and $\|\cdot\|_{2}$ are collinear in $C-C$

(2) For each $y \in C$ the norms $\|\cdot\|_{1}$ and $\|\cdot\|_{2}$ are collinear in $C-y$.

Proof. It is clear that (1) implies (2); thus we are going to show that (2) implies (1). We may suppose without loss of generality that $C$ have at least two elements. For each $y \in C$ we define $\lambda_{y}>0$ as the scalar such that

$$
\|x-y\|_{1}=\lambda_{y}\|x-y\|_{2} \quad \forall x \in C .
$$

Let $y_{0} \in C$; then for all $x \in C$ we have that $\left\|x-y_{0}\right\|_{1}=$ $\lambda_{y_{0}}\left\|x-y_{0}\right\|_{2}$ and $\left\|x-y_{0}\right\|_{1}=\lambda_{x}\left\|x-y_{0}\right\|_{2}$; since $C$ has more than one element we have $\lambda_{x}=\lambda_{y_{0}}$ for all $x \in C$; that is $\|x-y\|_{1}=\lambda_{y_{0}}\|x-y\|_{2}$ for all $x, y \in C$ and then $\|\cdot\|_{1}$ and $\|\cdot\|_{2}$ which conclude the proof.

Definition 16. Given normed spaces $X_{1}, X_{2}, Y_{1}$, and $Y_{2}$ and for $i=1,2, C_{i} \subset X_{i}$, and $D_{i} \subset Y_{i}$ being convex, one calls that $T: C_{1} \rightarrow C_{2}$ and $S: D_{1} \rightarrow D_{2}$ are isometrically affine equivalent, in symbols $T \simeq S$, if affine surjective isometries $\phi_{i}: C_{1} \rightarrow D_{i}$ for $i=1,2$ exist, such that $T=\phi_{2}^{-1} S \phi_{1}$ and $S=\phi_{2} T \phi_{1}^{-1}$.

The intuition is that two operators are isometrically affine equivalent if they have essentially the same transformation.

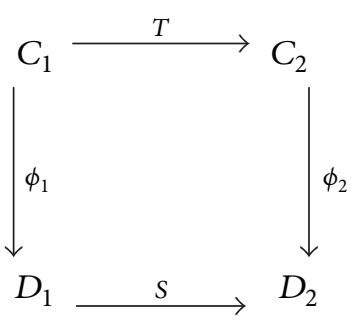

The following lemma summarizes some properties of the isometrically affine relation.

Lemma 17. Let $T \simeq S$; then one has the following.

(1) $T$ is continuous if and only if $S$ is continuous; moreover $T$ and $S$ have the same modulus of continuity $\omega(t)$; hence the affine isometry relation preserves uniform continuity and the Lipschitzian property with the same Lipschitz constant.

(2) $T$ is affine if and only if $S$ is affine.

The previous lemma allows us to translate properties of real functions to functions defined between arbitrary Banach spaces; this provides us with elements to ensure the existence of operators with desired properties.

Theorem 18. Let $(X,\|\cdot\|)$ be a normed space, $C$ be a nontrivial convex subset of $X$, and $f$ be a function; then we have the following.

(1) If $f \simeq g$ with $g:([0,1],|\cdot|) \rightarrow([0,1],|\cdot|)$ is Lipschitzian and $x, y \in C$ and $x \neq y$, then $T: C \rightarrow C$ exists as Lipschitzian such that $K(T,\|\cdot\|)=K(f,|\cdot|)$ and $\left.T\right|_{[x, y]} \simeq f$. 
(2) If $f \simeq g$ with $g:([0,1],|\cdot|) \rightarrow(\mathbb{R},|\cdot|)$ is Lipschitzian, then $x, y \in C, x \neq y$, and $T: C \rightarrow C$ exist as Lipschitzian such that $K(T,\|\cdot\|)=K(f,|\cdot|)$ and $\left.T\right|_{[x, y]} \simeq f$.

Proof. We suppose the hypothesis of (1). Since $C$ is convex, then $[x, y] \subset C$, and since all norms in $\mathbb{R}$ are collinear, then the Lipschitz constant $k$ of $g$ is independent of the norm in $\mathbb{R}$.

We consider the operator $\phi:[0,1] \rightarrow[x, y]$ defined by $\phi r=r x+(1-r) y$ for each $r \in[0,1]$ and we provide the metric space $[0,1]$ with the norm $|r|_{0}=\|x-y\||r|$ for each $r \in[0,1]$. It is concluded from definition that $\left([0,1],|\cdot|_{0}\right)$ is $\phi$ isometric to the space $([x, y],\|\cdot\|)$. It is known that $\left([0,1],|\cdot|_{0}\right)$ is a metric space with the binary intersection property and metrically convex; then $([x, y],\|\cdot\|)$ have the same properties.

We call $h:[x, y] \rightarrow[x, y]$ the operator defined by $h=$ $\phi g \phi^{-1}$; hence $h \simeq g$ and $g \simeq f$; thus by Lemma 17 we have $K(h,\|\cdot\|)=k$; then by Definition 1.3 and Proposition 1.4 of [15], an extension $T: C \rightarrow[x, y] \subset C$ of $h$ exists with $K(T,\|\cdot\|)=K(h,\|\cdot\|)$; then $\left.T\right|_{[x, y]} \simeq f$ and for Lemma $17 T$ and $f$ have the same Lipschitz constant.

Now we are going to prove (2). Let $f$ be a function such that $g$ exists as above; then optimal $r, s \in \mathbb{R}$ exist such that $g([0,1]) \subset[r, s] \subset \mathbb{R}$. There are two possibilities:

$$
\begin{gathered}
s-r \leq 1 \\
\text { or } s-r>1 .
\end{gathered}
$$

If $s-r \leq 1$ then we can choose $r^{\prime} \leq r \leq s \leq s^{\prime}$ such that $s^{\prime}-r^{\prime}=1$; thus $[r, s] \subset\left[r^{\prime}, s^{\prime}\right]$; we define the operator $\phi$ : $([0,1],|\cdot|) \rightarrow\left(\left[r^{\prime}, s^{\prime}\right],|\cdot|\right)$ by $\phi t=t r^{\prime}+(1-t) s^{\prime}$; it is clear that $\phi$ is an isometry; then the operator $h=\phi^{-1} g=\phi^{-1} g I d_{[0,1]}$ is Lipschitzian and satisfies $h \simeq g \simeq f$ which is the hypothesis of (1); thus without loss of generality, we may suppose that $s-r>1$.

We fix distinct $x, y \in C$, for each $t \in[0,1]$; we call $a_{t}=$ $t r+(1-t) s$ and define a norm on $[r, s]$ by $\left|a_{t}-a_{p}\right|_{0}=\| x-$ $y \||t-p|$ for each $t, p \in[0,1]$; we chose $a, b \in[r, s]$ such that $a<b$ and $b-a=1$, and we define a norm on $[0,1]$ by $|t|_{1}=|a-b|_{0}|t|$ for each $t \in[0,1]$; we consider the operators $\phi_{2}:\left([r, s],|\cdot|_{0}\right) \rightarrow([x, y],\|\cdot\|)$ defined by $\phi_{2}(t r+(1-t) s)=$ $t x+(1-t) y$ and $\phi_{1}:\left([0,1],|\cdot|_{1}\right) \rightarrow\left(\left[\phi_{2}(a), \phi_{2}(b)\right],\|\cdot\|\right)$ defined by $\phi_{1}(t)=t \phi_{2}(a)+(1-t) \phi_{2}(b)$ for each $t \in[0,1]$ which are surjective affine isometries.

Thus the operator $h=\phi_{2} g \phi_{1}^{-1}$ satisfies $h:\left[\phi_{2}(a)\right.$, $\left.\phi_{2}(b)\right] \rightarrow[x, y]$ and $h \simeq g \simeq f$; then by Lemma $17 h$ and $f$ have the same Lipschitz constant $k$ and again by Definition 1.3 and Proposition 1.4 of [15], an extension $T: C \rightarrow[x, y] \subset C$ of $h$ exists with the same Lipschitz constant.

Corollary 19. Let $(X,\|\cdot\|)$ be a normed space and $C \subset X$ be a nontrivial convex; then a nonaffine operator always exists in $N E(C,\|\cdot\|)$.

Proof. Let $f \in \operatorname{Lip}([0,1])$ be a nonaffine function such that $K(f,|\cdot|) \leq 1$ and distinct $x, y \in C$; then by Theorem 18 , an operator $T: C \rightarrow C$ exists with $K(T,\|\cdot\|)=K(f,|\cdot|) \leq 1$ and $\left.T\right|_{[x, y]} \simeq f$; thus by Lemma $17 T$ is a nonaffine operator.

From Remark 13 given a nonempty subset $C$ of a normed space $X$ and two collinear norms $\|\cdot\|_{1}$ and $\|\cdot\|_{2}$ in $C-C$, we have that $N E\left(C,\|\cdot\|_{1}\right)=N E\left(C,\|\cdot\|_{2}\right)$. Now, we prove the reciprocal of the previous statement.

Remark 20. It is important to note that the collinearity of two norms $\|\cdot\|$ and $\|\cdot\|_{1}$ on $C$ does not necessarily imply that, for each $T: C \rightarrow C, K(T,\|\cdot\|)=K\left(T,\|\cdot\|_{1}\right)$.

Let $\|\cdot\|_{2}$ and $\|\cdot\|_{1}$ be, respectively, the euclidean and the one norm on $\mathbb{R}^{2}$; then we define

$$
\|(x, y)\|= \begin{cases}\|(x, y)\|_{2}, & \text { if } \operatorname{sign}(x y)=1 \\ \|(x, y)\|_{1}, & \text { if } \operatorname{sign}(x y)=-1\end{cases}
$$

for each $(x, y) \in \mathbb{R}^{2}, C=\left\{(x, y) \mid\|(x, y)\|_{2} \leq 1\right\}$, and $S$ the rotation on $\mathbb{R}^{2}$ determined by the matrix $\left(\begin{array}{cc}\cos (\pi / 2) & -\sin (\pi / 2) \\ \sin (\pi / 2) & \cos (\pi / 2)\end{array}\right)$. It is clear that $S(C) \subset C, K\left(S,\|\cdot\|_{2}\right)=1$, and $K(S,\|\cdot\|)>1$; since $\|\cdot\|_{2}$ and $\|\cdot\|$ are collinear in the first quadrant, then they are collinear on $C+(1,1)$. Let $T=S+(1,1)$; then $T$ : $C+(1,1) \rightarrow C+(1,1)$ with $\|\cdot\|_{2}$ and $\|\cdot\|$ collinear on $C+(1,1)$, but $K\left(T,\|\cdot\|_{2}\right)=K\left(S,\|\cdot\|_{2}\right) \neq K(S,\|\cdot\|)=K(T,\|\cdot\|)$.

Theorem 21. Let $X$ be a normed space and $C$ a nontrivial convex subset of $X$. If $\|\cdot\|_{1},\|\cdot\|_{2} \in \mathfrak{N}(X)$, then the following statements are equivalent.

(1) $\|\cdot\|_{1}$ and $\|\cdot\|_{2}$ are not collinear in $C-C$.

(2) $\operatorname{NE}\left(C,\|\cdot\|_{1}\right) \neq N E\left(C,\|\cdot\|_{2}\right)$.

(3) $T \in N E\left(C,\|\cdot\|_{1}\right) \backslash N E\left(C,\|\cdot\|_{2}\right)$ and $S \in N E\left(C,\|\cdot\|_{2}\right) \backslash$ $N E\left(C,\|\cdot\|_{1}\right)$ exist.

(4) Nonaffine mappings $T, S$ with $T \in N E\left(C,\|\cdot\|_{1}\right) \backslash$ $N E\left(C,\|\cdot\|_{2}\right)$ and $S \in N E\left(C,\|\cdot\|_{2}\right) \backslash N E\left(C,\|\cdot\|_{1}\right)$ exist.

Proof. It is clear that we only need to prove that (1) implies (4). We suppose (1); then by Lemma $15 y \in C$ exists such that $\|\cdot\|_{1}$ and $\|\cdot\|_{2}$ are not collinear in $C-y$; thus without loss of generality we may assume that $0 \in C$.

Since $\|\cdot\|_{1}$ and $\|\cdot\|_{2}$ are not collinear in $C$ and $0 \in C$, then by Lemma $14 x, y \in C \backslash\{0\}$ exist such that

$$
\begin{gathered}
\|x\|_{1}=\|y\|_{1}, \\
\|x\|_{2}<\|y\|_{2} .
\end{gathered}
$$

Without loss of generality we may assume that $\|x\|_{1}=\|y\|_{1}=$ 1.

Let $\alpha_{0} \in\left(\|x\|_{2} /\|y\|_{2}, 1\right)$; then $z=\alpha_{0} y$ satisfies $\|x\|_{2}<$ $\|z\|_{2}<\|y\|_{2}$; we define the operator $f:[0, x] \rightarrow[0, y]$ by

$$
f(\alpha x)= \begin{cases}\alpha y, & \text { if } 0 \leq \alpha \leq \alpha_{0} \\ z, & \text { if } \alpha_{0} \leq \alpha \leq 1\end{cases}
$$


Since

$$
\begin{aligned}
f\left(\left(1-\alpha_{0}\right) 0+\alpha_{0} x\right) & =z=\alpha_{0} y \neq \alpha_{0}\left(\alpha_{0} y\right) \\
& =\left(1-\alpha_{0}\right) 0+\alpha_{0} f(x) \\
& =\left(1-\alpha_{0}\right) f(0)+\alpha_{0} f(x),
\end{aligned}
$$

then $f$ is nonaffine.

We affirm that $K\left(f,\|\cdot\|_{1}\right)=1$; in fact, let $\alpha, \beta \in[0,1]$; then by symmetry we only have the following three cases.

(i) $0 \leq \alpha, \beta \leq \alpha_{0}$; then

$$
\begin{aligned}
\|f(\alpha x)-f(\beta x)\|_{1} & =\|\alpha y-\beta y\|_{1}=|\alpha-\beta|\|y\|_{1} \\
& =|\alpha-\beta|\|x\|_{1}=\|\alpha x-\beta x\|_{1} .
\end{aligned}
$$

(ii) $\alpha_{0} \leq \alpha, \beta \leq 1$, then $\|f(\alpha x)-f(\beta x)\|_{1}=\|z-z\|_{1}=$ $0 \leq\|\alpha x-\beta x\|_{1}$.

(iii) $0 \leq \alpha<\alpha_{0}<\beta \leq 1$; then

$$
\begin{aligned}
\|f(\alpha x)-f(\beta x)\|_{1} & =\|\alpha y-z\|_{1}=\left\|\alpha y-\alpha_{0} y\right\|_{1} \\
& =\left|\alpha-\alpha_{0}\right|\|y\|_{1}=\left|\alpha-\alpha_{0}\right|\|x\|_{1} \\
& <|\alpha-\beta|\|x\|_{1}=\|\alpha x-\beta y\|_{1} .
\end{aligned}
$$

Now we prove that $K\left(f,\|\cdot\|_{2}\right)>1$; for this we have

$$
\begin{aligned}
\|f(0)-f(x)\|_{2} & =\|0-z\|_{2}=\|z\|_{2}>\|x\|_{2} \\
& =\|0-x\|_{2} .
\end{aligned}
$$

Since $\|x\|_{1}=\|y\|_{1}=1$, then the sets $[0, x]$ and $[0, y]$ are affine $\|\cdot\|_{1}$-isometric; then by Remark 6 , they are affine isometric to $([0,1],|\cdot|)$ and $g:([0,1],|\cdot|) \rightarrow([0,1],|\cdot|)$ exists with $f \simeq g$; thus by Theorem 18 an extension $T: C \rightarrow[0, y] \subset C$ of $f$ exists, with the same $\|\cdot\|_{1}$-Lipschitz constant that means $K\left(T,\|\cdot\|_{1}\right)=1$; thus $T \in N E\left(C,\|\cdot\|_{1}\right)$, but $T$ is $\|\cdot\|_{2}$-Lipschitz and $K\left(T,\|\cdot\|_{2}\right) \geq K\left(f,\|\cdot\|_{2}\right)>1$. Then $T \notin N E\left(C,\|\cdot\|_{2}\right)$.

In a similar way we prove the existence of $S \in N E(C$, $\left.\|\cdot\|_{2}\right) \backslash N E\left(C,\|\cdot\|_{1}\right)$, by exchanging the order of the norms and applying Lemma 14.

Remark 22. We notice that given a convex subset $C$ that lies inside a one-dimensional affine subspace of $X$, then for each pair of norms $\|\cdot\|_{1}$ and $\|\cdot\|_{2}$ in $X$ not necessarily equivalent, we have that $N E\left(C,\|\cdot\|_{1}\right)=N E\left(C,\|\cdot\|_{2}\right)$; this is because $C$ is affine isometric to a closed convex subset of $\mathbb{F}$ for a norm, and all norms in $\mathbb{F}$ are collinear; then by Theorem 21 the conclusion follows.

Corollary 23. Let $X$ be a normed space and $C \subset X$ such that $x, y \in C$ exist so that for each $z \in C \alpha \in \mathbb{F}$ exists with $z=$ $\alpha x+(1-\alpha) y$; then, for each norm $\|\cdot\|$ on $X, \mathcal{S}^{\prime}(C)=N E(C,\|\cdot\|)$.

Remark 24. From the last theorem we infer that it is not possible to compare the families of nonexpansive mappings under noncollinear renormings with respect to the contention relation because, under noncollinear renorming, nonexpansive mappings always win and lose.
Corollary 25. Let $X$ be a normed space, and $C \subset X$ such that for each $x, y \in C z \in C$ exists with $z \neq \alpha x+(1-\alpha) y$ for each $\alpha \in \mathbb{F}$; then for each norm $\|\cdot\|$ on $X, N E(C,\|\cdot\|) \neq \mathcal{S}^{\prime}(C)$.

Corollary 26. The nonexpansive operators constructed in the proof of Lemma 15 do not belong to $S^{\prime}(C)$.

\section{Examples}

In the present section we support the theoretical results of the previous ones by providing some examples.

In general, given a Banach space $X$ and a convex subset $C$ of $X$, it is hard to construct examples of nontrivial nonexpansive operators from $C$ to $C$; by trivial we understand the elements in the sets $\delta(C), \mathcal{S}^{\prime}(C)$ or more general affine mappings from $C$ to itself. In this sense, when a new Banach space is studied to know some of its geometric properties, for instance, the FPP, then it is natural to ask for the existence of some nontrivial nonexpansive operators defined in that space. An example of last situation is the renorming $\|\cdot\|_{L}$ of $\ell_{1}$ studied by Lin [1], for which at the moment in the literature only few examples of nonexpansive mappings exist.

Example 27. Let $\left(\ell_{1},\|\cdot\|_{1}\right)$ be the Banach space of absolutely summable series of real numbers endowed whit the norm $\left\|\left(x_{n}\right)\right\|_{1}=\sum_{n=0}^{\infty}\left|x_{n}\right|$. It is well known that $\left(\ell_{1},\|\cdot \mid\|_{1}\right)$ does not have the FPP; the classical example is the right-shift operator $R$ defined from $C$ to $C$, where

$$
\begin{aligned}
C & =\overline{\operatorname{conv}\left\{e_{n} \mid n \in \mathbb{N}\right\}}{ }^{\|\cdot\|_{1}}, \\
R\left(x_{1}, x_{2}, \ldots\right) & =\left(0, x_{1}, \ldots\right) .
\end{aligned}
$$

For each $k \in \mathbb{N}$ let $v_{k}$ be the seminorm in $\ell_{1}$ defined by $\nu_{k}\left(\left(x_{n}\right)\right)=\sum_{n=k}^{\infty}\left|x_{n}\right|$; thus the $\|\cdot\|_{L}$ norm in $\ell_{1}$ is defined by

$$
\left\|\left(x_{n}\right)\right\|_{L}=\sup _{k} \gamma_{k} \nu_{k}\left(\left(x_{n}\right)\right) \quad \text { where } \gamma_{k}=\frac{8^{k}}{1+8^{k}} \text {. }
$$

Now we construct some examples of nontrivial $\|\cdot\|_{L^{-}}$ nonexpansive operators defined from $C$ to itself such that they are not $\|\cdot\|_{1}$-nonexpansive and the opposite.

Let

$$
\begin{aligned}
& g_{1}=e_{1}, \\
& g_{2}=\left(1-\frac{\gamma_{1}}{\gamma_{2}}\right) e_{1}+\frac{\gamma_{1}}{\gamma_{2}} e_{2}, \\
& g_{3}=\left(1-\frac{\gamma_{1}}{\gamma_{2}}\right) e_{1}+\left(\frac{\gamma_{1}}{\gamma_{2}}-\frac{\gamma_{1}}{\gamma_{3}}\right) e_{2}+\frac{\gamma_{1}}{\gamma_{3}} e_{3} ;
\end{aligned}
$$

then $\left\|g_{1}\right\|_{1}=\left\|g_{2}\right\|_{1}=\left\|g_{3}\right\|_{1}=1$ and $g_{1}, g_{2}, g_{3} \in C$.

As in Lemma 15, we consider the set $C-g_{2}$ and define

$$
\begin{aligned}
& x^{\prime}=g_{1}-g_{2}=\frac{\gamma_{1}}{\gamma_{2}}\left(e_{1}-e_{2}\right), \\
& y^{\prime}=g_{3}-g_{2}=\frac{\gamma_{1}}{\gamma_{3}}\left(e_{3}-e_{2}\right) ;
\end{aligned}
$$


then $\left\|x^{\prime}\right\|_{1}=2\left(\gamma_{1} / \gamma_{2}\right)$ and $\left\|y^{\prime}\right\|_{1}=2\left(\gamma_{1} / \gamma_{3}\right)$; since $2 \gamma_{1} \geq$ $\gamma_{2}, 2 \gamma_{2} \geq \gamma_{3}$ and $\gamma_{1} \neq \gamma_{2}$ we have

$$
\begin{aligned}
& \left\|x^{\prime}\right\|_{L}=\gamma_{1} 2 \frac{\gamma_{1}}{\gamma_{2}}=\gamma_{1}\left\|x^{\prime}\right\|_{1}, \\
& \left\|y^{\prime}\right\|_{L}=\max \left\{\gamma_{1} 2 \frac{\gamma_{1}}{\gamma_{3}}, \gamma_{2} 2 \frac{\gamma_{1}}{\gamma_{3}}, \gamma_{1}\right\}=2 \gamma_{1} \frac{\gamma_{2}}{\gamma_{3}}=\gamma_{2}\left\|y^{\prime}\right\|_{1} ;
\end{aligned}
$$

hence $\|\cdot\|_{1}$ and $\|\cdot\|_{L}$ are not collinear in $C-g_{2}$; in fact, by Lemma 15, they are not collinear in $C-C$.

Now we make the construction of Lemma 14 in this particular case. It is clear that

$$
\frac{\left\|x^{\prime}\right\|_{1}}{\left\|x^{\prime}\right\|_{L}}=\frac{1}{\gamma_{1}}>\frac{1}{\gamma_{2}}=\frac{\left\|y^{\prime}\right\|_{1}}{\left\|y^{\prime}\right\|_{L}} .
$$

Since $\gamma_{3} \geq \gamma_{2}$, then $\left\|y^{\prime}\right\|_{1} \leq\left\|x^{\prime}\right\|_{1}$; thus we define $x=y^{\prime}$ and $y=\left(\left\|y^{\prime}\right\|_{1} /\left\|x^{\prime}\right\|_{1}\right) x^{\prime} ;$ then by $(46)$

$$
\begin{aligned}
& \|x\|_{1}=\|y\|_{1}, \\
& \|y\|_{L}=\frac{\left\|y^{\prime}\right\|_{1}}{\left\|x^{\prime}\right\|_{1}}\left\|x^{\prime}\right\|_{L}<\left\|y^{\prime}\right\|_{L}=\|x\|_{L} .
\end{aligned}
$$

We get $\alpha_{0} \in\left(\|y\|_{L} /\|x\|_{L}, 1\right)$; thus $z=\alpha_{0} x \in[0, x]$ and $\|y\|_{L}<$ $\|z\|_{L}<\|x\|_{L}$. We consider the function $f:[0, y] \rightarrow[0, x]$ defined by

$$
f(\alpha y)= \begin{cases}\alpha x, & \text { if } 0 \leq \alpha \leq \alpha_{0}, \\ z, & \text { if } \alpha_{0} \leq \alpha \leq 1\end{cases}
$$

then by the same argument of the proof of Theorem 21, the existence of a nonaffine mapping $T \in N E\left(C,\|\cdot\|_{1}\right) \backslash N E(C$, $\left.\|\cdot\|_{L}\right)$ follows.

Now we prove the existence of a nonaffine mapping $S \in$ $N E\left(C,\|\cdot\|_{L}\right) \backslash N E\left(C,\|\cdot\|_{1}\right)$. From (46) it follows that

$$
\frac{\left\|x^{\prime}\right\|_{L}}{\left\|x^{\prime}\right\|_{1}}=\gamma_{1}<\gamma_{2}=\frac{\left\|y^{\prime}\right\|_{L}}{\left\|y^{\prime}\right\|_{1}} .
$$

Since $\gamma_{1} / \gamma_{2} \leq \gamma_{2} / \gamma_{3}$, then $\left\|x^{\prime}\right\|_{L} \leq\left\|y^{\prime}\right\|_{L}$; hence we define $x=x^{\prime}$ and $y=\left(\left\|x^{\prime}\right\|_{L} /\left\|y^{\prime}\right\|_{L}\right) y^{\prime}$; thus by (49)

$$
\begin{aligned}
\|x\|_{L} & =\|y\|_{L}, \\
\|y\|_{1} & =\frac{\left\|x^{\prime}\right\|_{L}}{\left\|y^{\prime}\right\|_{L}}\left\|y^{\prime}\right\|_{1}<\left\|x^{\prime}\right\|_{1}=\|x\|_{1} .
\end{aligned}
$$

Let $\lambda_{0} \in\left(\|y\|_{1} /\|x\|_{1}, 1\right)$; hence $w=\lambda_{0} x$ satisfies $\|y\|_{1}<$ $\|w\|_{1}<\|x\|_{1}$; we define the function $g:[0, y] \rightarrow[0, x]$,

$$
g(\lambda y)= \begin{cases}\lambda x, & \text { if } 0 \leq \lambda \leq \lambda_{0} \\ w, & \text { if } \lambda_{0} \leq \lambda \leq 1\end{cases}
$$

then again by the same argument of the proof of Theorem 21, we ensure the existence of a nonaffine mapping $S \in N E(C$, $\left.\|\cdot\|_{L}\right) \backslash N E\left(C,\|\cdot\|_{1}\right)$.
Remark 28. Let $\gamma=\left(\gamma_{k}\right)$ be a nondecreasing sequence in $(0,1)$ such that $\gamma_{k} \rightarrow 1$; then by [7], the renorming

$$
\left\|\left(x_{n}\right)\right\|_{\gamma}=\sup _{k} \gamma_{k} v_{k}\left(\left(x_{n}\right)\right)
$$

of $\left(\ell_{1},\|\cdot\|_{1}\right)$ has the FPP. Note that the previous example is valid for each renorming $\|\cdot\|_{\gamma}$ in which

(i) $\gamma_{1} \neq \gamma_{2}$,

(ii) $2 \gamma_{1} \geq \gamma_{2}$ and $2 \gamma_{2} \geq \gamma_{3}$,

(iii) $\gamma_{1} / \gamma_{2} \leq \gamma_{2} / \gamma_{3}$.

Example 29. Now we characterize the family of nonexpansive mappings $T: C \rightarrow C$ over any renorming, where $C$ is as in Example 27; that is, we characterize the set $\delta^{\prime}(C)$. The next calculations are effortless if we consider the equality

$$
C=\left\{\left(x_{n}\right) \in \ell_{1} \mid x_{n} \geq 0, \quad \sum x_{n}=1\right\} .
$$

It is clear that $C$ does not lie inside a one-dimensional affine subspace of $\ell_{1}$; then by Corollary 8 , for each $T \in \mathcal{S}^{\prime}(C),\left(y_{n}\right) \in$ $\ell_{1}$ and $|\lambda| \leq 1$ exist such that $T\left(x_{n}\right)=\left(y_{n}\right)+\lambda\left(x_{n}\right)$ for each $\left(x_{n}\right) \in C$.

We affirm that each entry of $\left(y_{n}\right)$ is nonnegative and $0 \leq$ $\lambda \leq 1$; for this, if $y_{k}<0$ for some $k \in \mathbb{N}$ then the $k$-entry of $T e_{k+1}$ is $y_{k}$; thus $T e_{k} \notin C$; hence for each $k \in \mathbb{N}$ we have $y_{k} \geq 0$. If $-1 \leq \lambda<0$, let $k \in \mathbb{N}$ such that $0 \leq y_{k}<-\lambda$; then the $k$-entry of $T e_{k}$ is $y_{k}+\lambda<0$; hence $0 \leq \lambda \leq 1$.

Let $\left(x_{n}\right) \in C$; then

$$
\begin{aligned}
1 & =\left\|T\left(x_{n}\right)\right\|_{1}=\left\|\left(y_{n}\right)+\lambda\left(x_{n}\right)\right\|=\sum\left|y_{n}+\lambda x_{n}\right| \\
& =\sum y_{n}+\sum \lambda x_{n}=\sum y_{n}+\lambda ;
\end{aligned}
$$

thus $0 \leq \lambda \leq 1$ and $\left(y_{n}\right) \in(1-\lambda) C$, which agrees with notation of Remark $1 \mathcal{S}^{\prime}(C) \subset\left\{T=\left(y_{n}\right)+\lambda I \mid 0 \leq \lambda \leq\right.$ 1 and $\left.\left(y_{n}\right) \in(1-\lambda) C\right\}$; the proof of the other contention is a direct calculation; thus

$$
\begin{aligned}
& \mathcal{S}^{\prime}(C) \\
& \quad=\left\{T=\left(y_{n}\right)+\lambda I \mid 0 \leq \lambda \leq 1,\left(y_{n}\right) \in(1-\lambda) C\right\} .
\end{aligned}
$$

In this case $\mathcal{S}(C)=\mathcal{S}^{\prime}(C)$; for this, let $0 \leq \lambda \leq 1$ and $\left(y_{n}\right) \in$ $(1-\lambda) C$; thus $(1-\lambda)^{-1}\left(y_{n}\right) \in C$; then $T=(1-\lambda)[(1-$ $\left.\lambda)^{-1}\left(y_{n}\right)\right]+\lambda I \in \mathcal{S}(C)$.

Example 30. Let $(X,\|\cdot\|)$ be a normed space and $B$ its unit ball. We characterize the set $\mathcal{S}^{\prime}(B)$. By Corollary 8 , for each $T \in \mathcal{S}^{\prime}(B), y \in X$ and $|\lambda| \leq 1$ exist with $T x=y+\lambda x$ for each $x \in B$; we affirm that $|\lambda| \leq 1$ and $y \in(1-|\lambda|) B$; in fact, let $|\lambda| \leq 1, y \in(1-|\lambda|) B$, and $x \in B$; then

$$
\begin{aligned}
\|T x\| & =\|y+\lambda x\| \leq\|y\|+|\lambda|\|x\| \leq(1-|\lambda|)+|\lambda| \\
& =1 ;
\end{aligned}
$$

hence, with notation of Remark 1, $\{T=y+\lambda I|| \lambda \mid \leq$ 1 and $y \in(1-|\lambda|) B\} \subset \mathcal{S}^{\prime}(B)$. Now we prove the other 
contention. Let $|\lambda| \leq 1$ and $y \notin(1-|\lambda|) B$; then $\|y\|>1-|\lambda|$; we define $x=\lambda^{-1}(|\lambda| /\|y\|) y$; thus $x \in B$; since

$$
\begin{aligned}
\|y+\lambda x\| & =\left\|y+\frac{|\lambda|}{\|y\|} y\right\|=\left(1+\frac{|\lambda|}{\|y\|}\right)\|y\| \\
& =\|y\|+|\lambda|>1-|\lambda|+|\lambda|=1,
\end{aligned}
$$

then $y+\lambda x \notin B$; hence

$$
\mathcal{S}^{\prime}(B)=\{T=y+\lambda I|| \lambda \mid \leq 1, y \in(1-|\lambda|) B\} .
$$

\section{Competing Interests}

The authors declare that there is no conflict of interests regarding the publication of this paper.

\section{Acknowledgments}

All authors were partially supported by CONACyT Project 243722. The first author was fully supported by CONACyT national grant.

\section{References}

[1] P.-K. Lin, "There is an equivalent norm on 11 that has the fixed point property," Nonlinear Analysis: Theory, Methods \& Applications, vol. 68, no. 8, pp. 2303-2308, 2008.

[2] T. Domínguez-Benavides, "A renorming of some nonseparable Banach spaces with the fixed point property," Journal of Mathematical Analysis and Applications, vol. 350, no. 2, pp. 525-530, 2009.

[3] P. N. Dowling, C. J. Lennard, and B. Turett, "Reflexivity and the fixed-point property for nonexpansive maps," Journal of Mathematical Analysis and Applications, vol. 200, no. 3, pp. 653662, 1996.

[4] A. Barrera-Cuevas and M. A. Japón, "New families of nonreflexive Banach spaces with the fixed point property," Journal of Mathematical Analysis and Applications, vol. 425, no. 1, pp. 349363, 2015.

[5] H. Fetter and B. Gamboa de Buen, "Banach spaces with a basis that are hereditarily asymptotically isometric to 11 and the fixed point property," Nonlinear Analysis: Theory, Methods \& Applications, vol. 71, no. 10, pp. 4598-4608, 2009.

[6] B. G. de Buen and F. Núñez-Medina, "A generalization of a renorming theorem by Lin and a new nonreflexive space with the fixed point property which is nonisomorphic to $\mathrm{l}_{1}$," Journal of Mathematical Analysis and Applications, vol. 405, no. 1, pp. 57-70, 2013.

[7] C. A. Linares and M. A. Japon, "A renorming in some Banach spaces with applications to fixed point theory," Journal of Functional Analysis, vol. 258, no. 10, pp. 3452-3468, 2010.

[8] C. A. Hernàndez-Linares and M. A. Japón, "Rays of equivalent norms with the fixed point property in some nonreflexive Banach spaces," Journal of Nonlinear and Convex Analysis, vol. 15, no. 2, pp. 355-377, 2014.

[9] C. A. Hernàndez-Linares, M. A. Japón, and E. Llorens-Fuster, "On the structure of the set of equivalent norms on $\ell_{1}$ with the fixed point property," Journal of Mathematical Analysis and Applications, vol. 387, no. 2, pp. 645-654, 2012.
[10] E. Llorens-Fuster, "The fixed point property for renormings of $l_{2}$," Arabian Journal of Mathematics, vol. 1, no. 4, pp. 511-528, 2012.

[11] A. Betiuk-Pilarska and T. Domínguez-Benavides, "The fixed point property for some generalized nonexpansive mappings and renormings," Journal of Mathematical Analysis and Applications, vol. 429, no. 2, pp. 800-813, 2015.

[12] E. Moreno-Gàlvez and E. Llorens-Fuster, "The fixed point property for some generalized nonexpansive mappings in a nonreflexive Banach space," Fixed Point Theory, vol. 14, no. 1, pp. 141-150, 2013.

[13] M. D. Kirszbraun, "Über die zusammenziehende und Lipschitzsche Transformationen," Fundamenta Mathematicae, vol. 22, no. 1, pp. 77-108, 1934.

[14] F. A. Valentine, "A Lipschitz condition preserving extension for a vector function," American Journal of Mathematics, vol. 67, pp. 83-93, 1945.

[15] Y. Benyamini and J. Lindenstrauss, Geometric Nonlinear Functional Analysis, vol. 48, American Mathematical Society, 1998. 


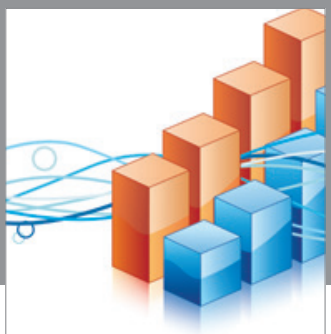

Advances in

Operations Research

vatem alat4

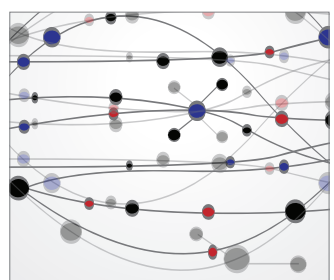

\section{The Scientific} World Journal
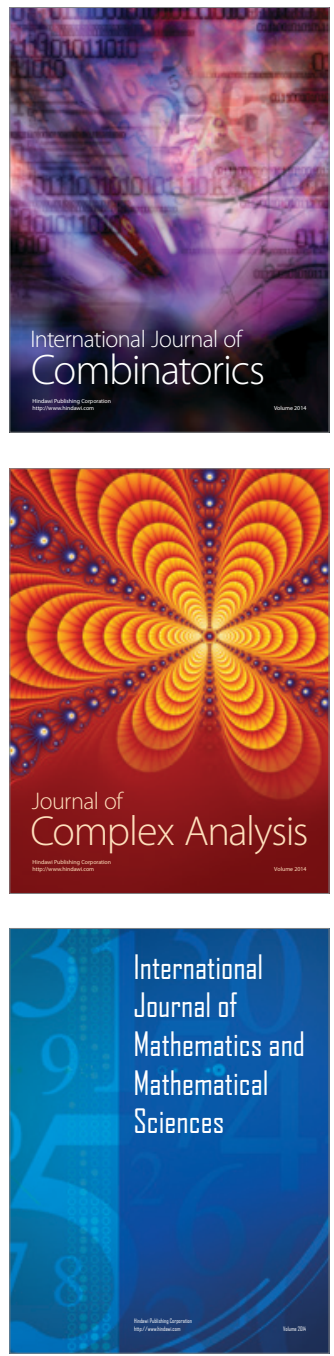
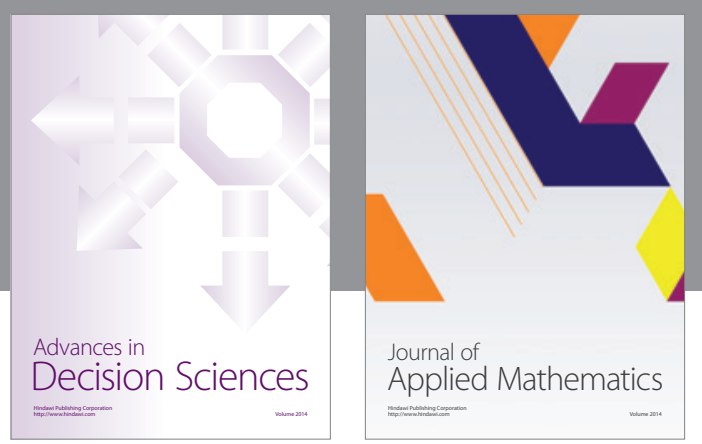

Algebra

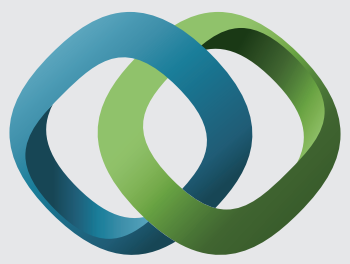

\section{Hindawi}

Submit your manuscripts at

http://www.hindawi.com
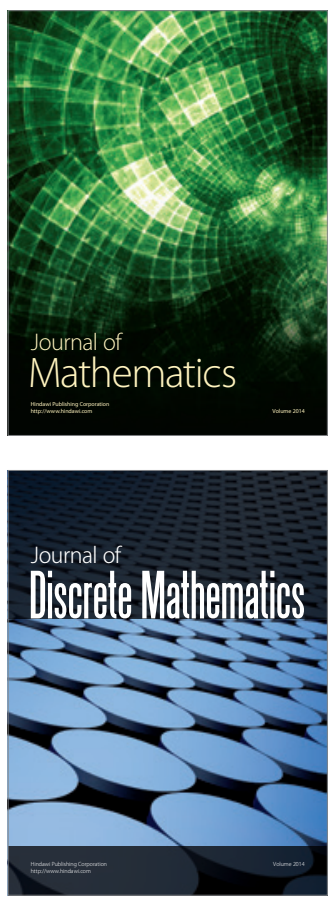

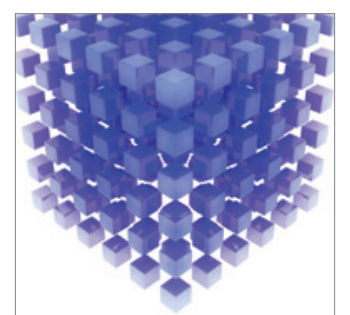

Mathematical Problems in Engineering
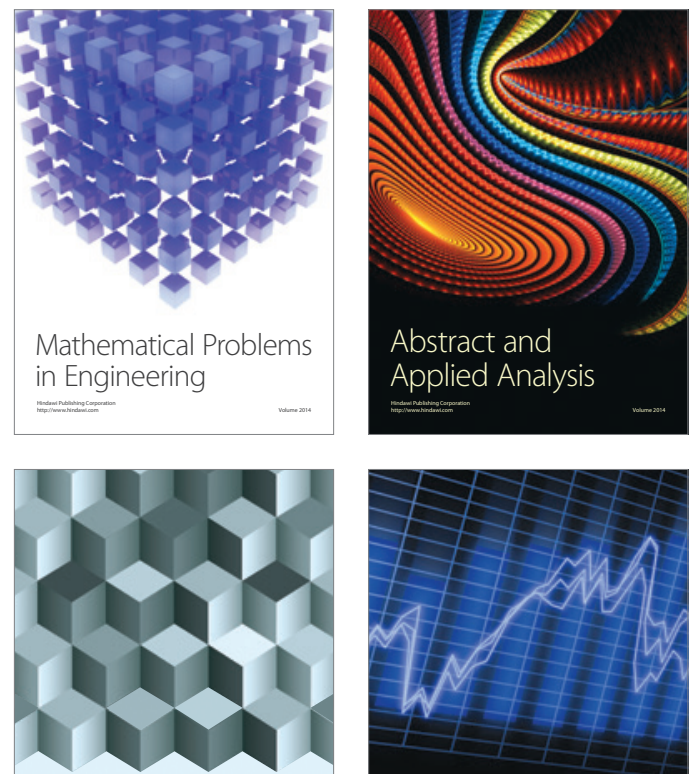

Journal of

Function Spaces

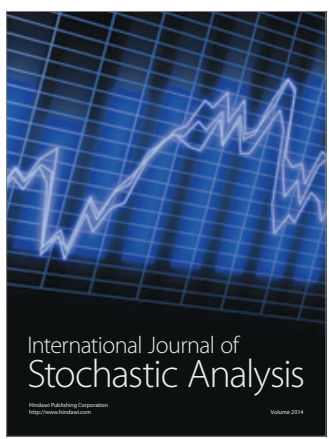

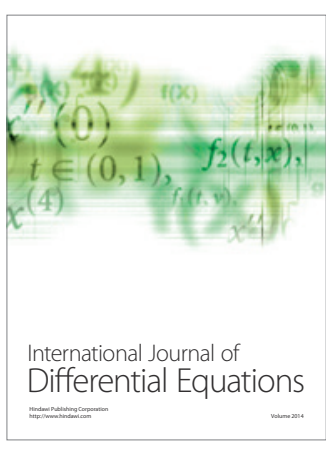
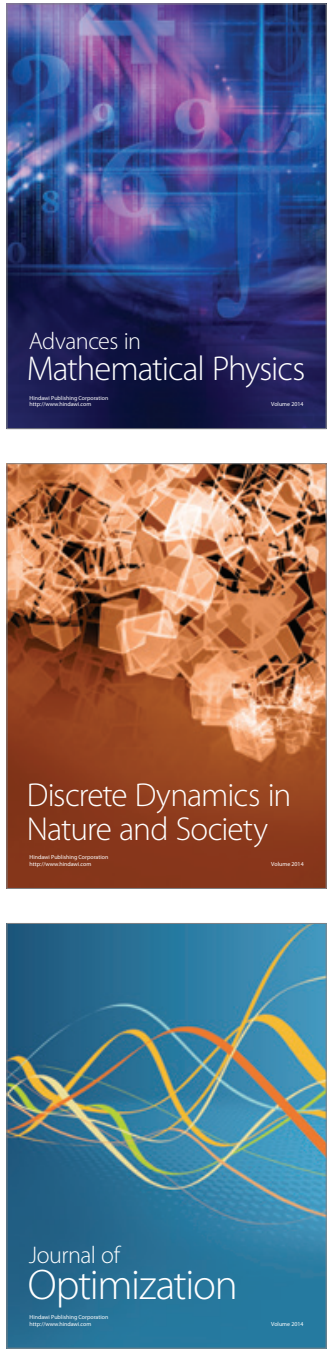